Check for updates

Cite this: DOI: 10.1039/c8sm02504e

\title{
Binding constant of membrane-anchored receptors and ligands that induce membrane curvatures
}

\author{
Long $\mathrm{Li}^{a}{ }^{a}$ Jinglei Hu, (D)*b Liang $\mathrm{Li}^{\mathrm{c}}$ and Fan Song $(\mathbb{D}$ *ad
}

\begin{abstract}
Cell adhesion is crucial for immune response, tissue formation, and cell locomotion. The adhesion process is mediated by the specific binding of membrane-anchored receptor and ligand proteins. These adhesion proteins are in contact with the membranes and may generate curvature, which has been shown for a number of membrane proteins to play an important role in membrane remodeling. An important question remains of whether the local membrane curvatures induced by the adhesion proteins affect their binding. We've performed Monte Carlo simulations of a mesoscopic model for membrane adhesion via the specific binding of curvature-inducing receptors and ligands. We find that the curvatures induced by the adhesion proteins do affect their binding equilibrium constant. We presented a theory that takes into account the membrane deformations and protein-protein interactions due to the induced curvatures, and agrees quantitatively with our simulation results. Our study suggests that the ability to induce membrane curvatures represents a molecular property of the adhesion proteins and should be carefully considered in experimental characterization of the binding affinity.
\end{abstract}

Received 11th December 2018, Accepted 10th March 2019

DOI: $10.1039 / \mathrm{c} 8 \mathrm{sm} 02504 \mathrm{e}$

rsc.li/soft-matter-journal

\section{Introduction}

Cell adhesion is pivotal for numerous cellular activities such as signal transduction, tissue formation, immune responses, and cell locomotion. ${ }^{1-3}$ The adhesion process is mediated by the specific binding of membrane-anchored receptor and ligand molecules, which is quantified by the two-dimensional (2D) binding equilibrium constant ${ }^{4-7}$

$$
K=\frac{[\mathrm{RL}]}{[\mathrm{R}][\mathrm{L}]},
$$

with the area concentrations of unbound receptors [R], unbound ligands $[\mathrm{L}]$, and receptor-ligand complexes [RL] given by the ratio of number of molecules $n_{\alpha}(\alpha=\mathrm{R}, \mathrm{L}$, or RL) to the area $A$ of the adhering zone. Various experimental techniques have been exploited to measure the binding equilibrium constant $K$, including fluorescence spectroscopy, ${ }^{8-11}$ atomic force microscopy, ${ }^{12-14}$ micropipette aspiration, ${ }^{15-17}$ and flow chamber. ${ }^{18-20}$

\footnotetext{
${ }^{a}$ State Key Laboratory of Nonlinear Mechanics (LNM) and Beijing Key Laboratory of Engineered Construction and Mechanobiology, Institute of Mechanics, Chinese Academy of Sciences, Beijing, 100190, China. E-mail: songf@lnm.imech.ac.cn

${ }^{b}$ Kuang Yaming Honors School \& Institute for Brain Sciences, Nanjing University, Nanjing, 210023, China. E-mail: hujinglei@nju.edu.cn

${ }^{c}$ School of Materials Science and Engineering, Wuhan Institute of Technology, Wuhan 430205, China

${ }^{d}$ School of Engineering Science, University of Chinese Academy of Sciences, Beijing, 100049, China
}

In contrast to soluble proteins, the binding of membraneanchored proteins has been shown to strongly depend on the membrane environment such as average membrane separation ${ }^{4,21-24}$ and membrane thermal roughness from shape fluctuations, ${ }^{6,11,21,22,25}$ and membrane nanosized domains enriched in saturated phospholipids and cholesterol that recruit the proteins. ${ }^{26-28}$ These studies help to identify important membrane properties impacting the binding of membrane adhesion proteins.

The adhesion proteins are associated with cell membranes via transmembrane domains (e.g. integrins, cadherins) or glycosylphosphatidyl-inositol anchors (e.g. CD48), and may very likely generate local membrane curvature. For instance, integrins $^{33-35}$ and the adhesion molecule CD44 $4^{36,37}$ were found to concentrate at such highly-curved membrane regions as filopodia. Extensive studies have shown that curvature-inducing proteins mainly include transmembrane proteins of wedge shape and peripheral proteins either binding to the surface of one membrane monolayer or inserting asymmetric amphipathic or hydrophobic structures into the bilayer. ${ }^{38-41}$ Crowding of monomeric hydrophilic protein domains bound to the membrane surface has been shown in vitro to induce curvature. ${ }^{42}$ Asymmetric adsorption or depletion layers of biomolecules has also been demonstrated to be an efficient way of inducing local membrane curvature. ${ }^{43,44}$ These mechanisms of membrane curvature generation can certainly be utilized by the adhesion proteins. The protein-induced curvature plays an important role in remodeling of both cell ${ }^{9,38,45-48}$ and model $^{39,49-54}$ membranes, but has been largely ignored in 
(a)

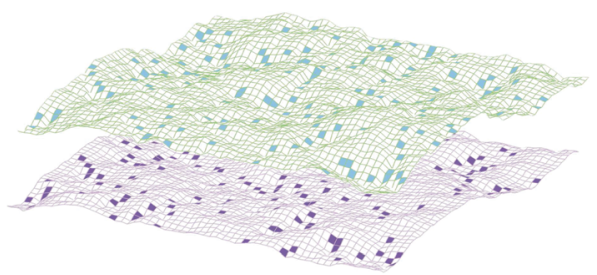

(b)

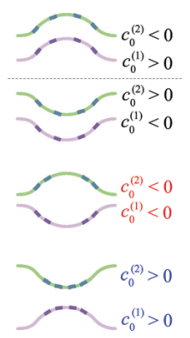

Fig. 1 (a) Simulation snapshot of two fluctuating membranes adhering via specific receptor-ligand binding. The receptor and ligand proteins are described by the light purple and blue patches, respectively. Each protein occupies a single membrane patch, diffuses along the membrane surface, and induces a local membrane curvature $c_{0}^{(1)}$ (receptors) or $c_{0}^{(2)}$ (ligands). A receptor binds to a ligand only when they are apposing to each other on the two membranes and within the binding range as specified in eqn (3). (b) Cartoon for different combinations of membrane curvatures $c_{0}^{(1)}$ and $c_{0}^{(2)}$ induced by the adhesion receptors and ligands. Note that the first two combinations are degenerate. By convention, proteins with positive curvature tend to induce outward bulging of the membrane patches away from the cytoplasm and negative inward. The local curvatures on each membrane induce repulsive interaction between the receptors or ligands, i.e., cis-repulsion. ${ }^{29-32}$

experimental and theoretical studies of cell adhesion. Its effect on the binding of membrane-anchored receptors and ligands remains to be understood.

In this article, we report Monte Carlo (MC) simulations of a statistical mechanical model for membrane adhesion via the specific binding of curvature-inducing receptor and ligand proteins with biologically relevant parameters. We simulated different combinations of protein-induced curvatures as shown in Fig. 1, and systematically varied the magnitude of the curvatures, the overall concentrations of the proteins as well as the bending rigidity of the protein-containing membrane segments. We find that the binding constant $K$ increases or decreases significantly with the protein-induced curvature depending on the combinations of the curvatures. To understand the curvature dependence of $K$, we've developed a theory in which the change of membrane deformations and protein interactions due to the protein-induced curvatures are taken into account by the distribution of local membrane separation and effective protein concentrations. The theory is in good quantitative agreement with our simulation results and provides a useful route to calculate $K$ for curvature-inducing receptors and ligands.

\section{Model and method}

The adhering membranes are represented by the two-dimensional square lattices ${ }^{55-58}$ as illustrated in Fig. 1. The overall energy of the adhesion system consists of the Helfrich elastic energy $\mathscr{H}_{\mathrm{el}}$ of the membranes and the interaction energy $\mathscr{H}_{\text {in }}$ of receptors and ligands. For tensionless membranes $\mathscr{H}_{\mathrm{el}}$ takes the form ${ }^{59}$

$$
\begin{aligned}
\mathscr{H}_{\mathrm{el}}= & \sum_{i} \sum_{j=1}^{2} \frac{\kappa_{i}^{(j)}}{2}\left\{\frac{1}{a^{2}}\left[\nabla_{\mathrm{d}}^{2} h_{i}^{(j)}-a^{2} n_{i}^{(j)} c_{0}^{(j)}\right]^{2}\right. \\
& \left.+\frac{1}{2}\left[n_{i}^{(j)} c_{0}^{(j)}\right]^{2}\left[\nabla_{\mathrm{d}} h_{i}^{(j)}\right]^{2}\right\}
\end{aligned}
$$

which sums over all the lattice sites or patches $i$ of the lower and upper membranes specified by $j=1,2$. The lattice size $a$ sets the length unit and can be mapped to $a=10 \mathrm{~nm}$ by matching the exclusion radius of membrane proteins. ${ }^{60} \kappa_{l}^{(j)}$ is the bending rigidity of each membrane patch, and $c_{0}^{(j)}$ the local membrane curvature induced by the anchored receptor $(j=1)$ or ligand $(j=2)$ proteins. The protein composition variable $n_{i}^{(j)}=1$ if the site $i$ of the lower (upper) membrane is occupied by a receptor (ligand), and $n_{i}^{(j)}=0$ otherwise. $h_{i}^{(j)}$ is the height of local membrane patch relative to the reference plane, and the difference $l_{i} \equiv h_{i}^{(2)}-h_{i}^{(1)}$ defines the separation of the two apposing patches at site $i . \nabla_{\mathrm{d}}^{2}$ is the discrete Laplacian operator. $\left[\nabla_{\mathrm{d}} h\right]^{2}$ results from Taylor expansion of the area measure $\mathrm{d} A=\left(1+\left[\nabla_{\mathrm{d}} h\right]^{2}\right)^{1 / 2} \mathrm{~d} x \mathrm{~d} y \approx\left(1+\frac{1}{2}\left[\nabla_{\mathrm{d}} h\right]^{2}\right) \mathrm{d} x \mathrm{~d} y \quad$ in the Monge parameterization of the membranes, and therefore describes the increase of membrane area per unit projected area. For tense membranes the energy term from the tension $\sigma$ is $\frac{\sigma}{2}\left[\nabla_{\mathrm{d}} h\right]^{2}$. One might then consider the term $\frac{\kappa_{i}^{(j)}}{4}\left[n_{i}^{(j)} c_{0}^{(j)}\right]^{2}$ as an additional effective tension acting on the membranes.

In the statistical model each receptor or ligand occupies a single membrane patch, diffuses along the membranes, and generates local membrane curvature $c_{0}^{(j)}$. Receptors and ligands at apposing patches $i$ of the two membranes bind via a squarewell potential with depth $U_{\mathrm{b}}>0$ and width $l_{\mathrm{b}}$, giving rise to the total interaction energy

$$
\mathscr{H}_{\text {in }}=-\sum_{i} \delta_{n_{i}^{(1)}, n_{i}^{(2)}} U_{\mathrm{b}} \theta\left(l_{\mathrm{b}} / 2-\left|l_{i}-l_{\mathrm{c}}\right|\right),
$$

where the Kronecker symbol $\delta_{n_{i}^{(1)}, n_{i}^{(2)}}=1$ for $n_{i}^{(1)}=n_{i}^{(2)}=1$, and $\delta_{n_{i}^{(1)}, n_{i}^{(2)}}=0$ otherwise. $l_{\mathrm{c}}$ is the length of receptor-ligand complexes. The Heaviside step function $\theta(\cdots)$ requires that the receptor and ligand in a complex should be within the binding range $l_{\mathrm{c}}-l_{\mathrm{b}} / 2 \leq l_{i} \leq l_{\mathrm{c}}+l_{\mathrm{b}} / 2$. Eqn (3) therefore effectively takes into account binding specificity of the receptor and ligand proteins.

We employ the standard Metropolis Monte Carlo (MC) method to simulate the adhesion systems in canonical ensemble with fixed number of receptors and ligands anchored to membranes of fixed area at constant temperature $T$. The membrane shape fluctuations are mimicked by the random vertical displacement of the lattices. The lateral diffusion of receptors and ligands is realized by a random jump of the molecule to one of the four nearest-neighboring patches. To capture the different physical time scales of the two motions, we performed on average $10 \mathrm{MC}$ steps for vertical displacement of all lattices every one MC step for random jump of all proteins during one MC cycle as done in ref. 27. We have simulated two adhering membranes each of projected area $A=L_{x} \times L_{y}=600 \times 600 \mathrm{~nm}^{2}$ under periodic boundary conditions. A relaxation run of $10^{6} \mathrm{MC}$ cycles is performed in each simulation for thermal equilibration and a subsequent run of $5 \times 10^{6} \mathrm{MC}$ cycles for statistical sampling. We then count the average number of free receptors, free ligands, and 

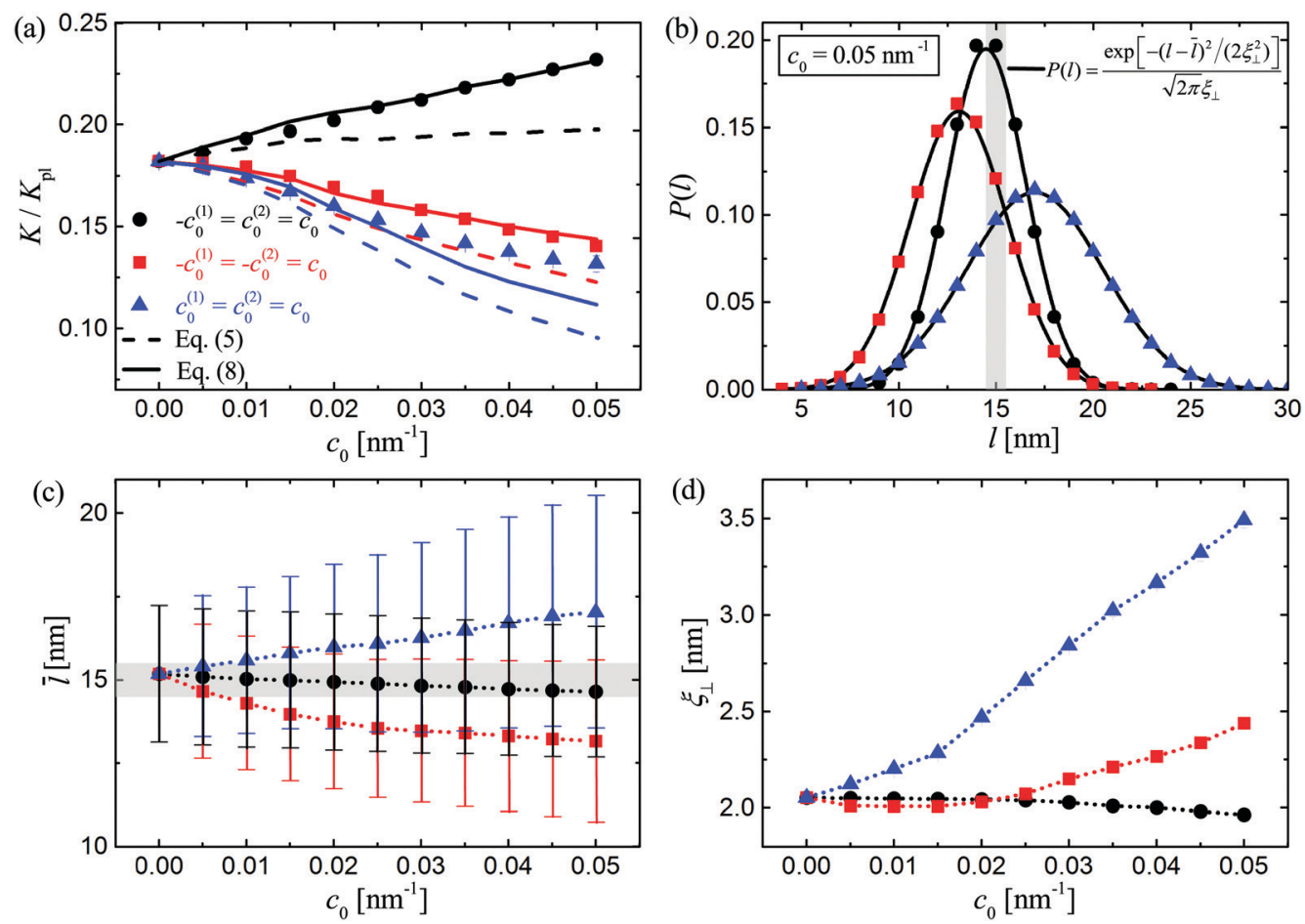

Fig. 2 Results for the binding of membrane-anchored receptor and ligand proteins that induce membrane curvature $\left|c_{0}^{(1)}\right|=\left|c_{0}^{(2)}\right|=c_{0}$. Data points in filled symbols are from MC simulations with $N_{\mathrm{R}}=N_{\mathrm{L}}=200$ and $\kappa=\kappa_{\mathrm{p}}=10 \mathrm{k}_{\mathrm{B}} T$. All the dotted lines are only guide for the eye. (a) Reduced binding constant $K / K_{\mathrm{pl}}$ vs. $c_{0} . K_{\mathrm{pl}} \equiv a^{2} \mathrm{e}^{\left.U_{\mathrm{b}} / K_{\mathrm{B}} T\right)}$ is the binding constant of the same receptor-ligand pair with $c_{0}=0$ and anchored to planar membranes at separation within the binding range. Dashed lines are theoretical results given by eqn (5) neglecting the curvature-induced protein-protein cis-repulsion. Solid lines are theoretical results from eqn (8). (b) Local separation l between apposing membrane patches obeys normal distribution $P(l)=\mathrm{e}^{-(l-\bar{l})^{2} /\left(2 \xi_{\perp}{ }^{2}\right)} /\left(\sqrt{2 \pi} \xi_{\perp}\right)$ with $\bar{l}$ the average separation and $\xi_{\perp}$ the relative membrane roughness. The shaded region indicates the binding range. Simulation data at $c_{0}=0.05 \mathrm{~nm}^{-1}$ is only shown here for clarity. (c) Average membrane separation $\bar{l} v s$. $c_{0}$. The vertical bars at each data point define the separation interval $\left[\bar{l}-\xi_{\perp}, \bar{l}+\xi_{\perp}\right]$. (d) Relative membrane roughness $\xi_{\perp}$ vs. $c_{0}$.

receptor-ligand complexes, and calculate the binding constant $K$ according to the definition in eqn (1).

The parameters in our simulations are chosen according to experiments or existing literature data. The bending rigidity $\kappa=10 k_{\mathrm{B}} T$ for the protein-free membrane patches, and $\kappa_{\mathrm{p}}=\kappa$ or $2 \kappa$ for the protein-containing patches. $k_{\mathrm{B}}$ is the Boltzmann constant. The protein-induced membrane curvature has a magnitude up to about $0.05 \mathrm{~nm}^{-1},{ }^{40,43,61-63}$ corresponding to the length scale four times of membrane thickness $\sim 5 \mathrm{~nm}$. There are three physically different combinations of membrane curvatures $c_{0}^{(1)}$ and $c_{0}^{(2)}$ induced by receptors and ligands as shown in Fig. 1(b). Without loss of generality we considered the cases with protein-induced curvatures $\left|c_{0}^{(1)}\right|=\left|c_{0}^{(2)}\right|=c_{0}$ for $0 \leq c_{0} \leq 0.05 \mathrm{~nm}^{-1}$. The binding strength $U_{\mathrm{b}}=5 k_{\mathrm{B}} T$, binding range $l_{\mathrm{b}}=1 \mathrm{~nm}$, and complex length $l_{\mathrm{c}}=15 \mathrm{~nm}{ }^{6,55}$ Given the membrane size, the number of receptors and ligands in each membrane is chosen to be $N_{\mathrm{R}}=N_{\mathrm{L}}=100,200$ or 400 . These values cover the range of 250 to $1000 \mu \mathrm{m}^{-2}$ for the overall protein concentration in the cell-adhesion zone. ${ }^{9,64,65}$

\section{Results and discussion}

The membrane curvatures generated by the anchored receptor and ligand proteins alter the local separation and elastic deformation of the two adhering membranes, and thereby affect the binding. Fig. 2(a) shows the reduced binding constant $K / K_{\mathrm{pl}}$ as a function of protein-induced membrane curvature $c_{0}$. $K_{\mathrm{pl}} \equiv a^{2} \mathrm{e}^{U_{\mathrm{b}} /\left(k_{\mathrm{B}} T\right)}$ is the binding constant of the same receptorligand pair anchored to two planar membranes within the binding range for $c_{0}=0$. Depending on the signs of the induced curvatures, the binding constant $K$ increases or decreases with $c_{0}$ as illustrated by the simulation data points in filled symbols. Such trends can be qualitatively explained as follows from a simplified theoretical consideration by neglecting the curvatureinduced protein-protein cis-repulsion. $^{29-32}$

For receptors and ligands anchored to planar membranes with separation $l$, the binding constant is $K(l)=K_{\mathrm{pl}}$ for $l_{\mathrm{c}}-l_{\mathrm{b}} / 2 \leq$ $l \leq l_{\mathrm{c}}+l_{\mathrm{b}} / 2$ and $K(l)=0$ otherwise according to the binding potential specified in eqn (3). In the general case of receptors and ligands anchored to fluctuating membranes, averaging $K(l)$ over the distribution $P(l)$ of local separation for protein-containing membrane patches leads to the binding constant ${ }^{21,22}$

$$
K=\int K(l) P(l) \mathrm{d} l .
$$

The simulation results in Fig. 2(b) show that the local separation, regardless of the magnitude and signs of the induced curvatures, follows a Gaussian distribution $P(l)=\mathrm{e}^{-(l-\bar{l})^{2} /\left(2 \xi_{\perp}{ }^{2}\right)} /\left(\sqrt{2 \pi} \xi_{\perp}\right)$ 
with $\bar{l}$ the average separation and $\xi_{\perp}$ the standard deviation, i.e., relative membrane roughness that characterizes the amplitude of relative shape fluctuations of the adhering membranes due to thermal excitation and receptor-ligand complex formation. The binding constant given by eqn (4) then assumes

$$
\begin{aligned}
K & =\int_{l_{\mathrm{c}}-l_{\mathrm{b}} / 2}^{l_{\mathrm{c}}+l_{\mathrm{b}} / 2} K_{\mathrm{pl}} \mathrm{e}^{-(l-\bar{l})^{2} /\left(2 \xi_{\perp}^{2}\right)} /\left(\sqrt{2 \pi} \xi_{\perp}\right) \mathrm{d} l \\
& =\frac{1}{2} K_{\mathrm{pl}}\left[\operatorname{Erf}\left(\tilde{l}_{1}\right)-\operatorname{Erf}\left(\tilde{l}_{2}\right)\right]
\end{aligned}
$$

with $\operatorname{Erf}(\cdots)$ the error function, $\tilde{l}_{1} \equiv\left(l_{\mathrm{c}}+l_{\mathrm{b}} / 2-\bar{l}\right) /\left(\sqrt{2} \xi_{\perp}\right)$ and $\tilde{l}_{2} \equiv\left(l_{\mathrm{c}}-l_{\mathrm{b}} / 2-\bar{l}\right) /\left(\sqrt{2} \xi_{\perp}\right)$ the reduced lengths. The dashed lines in Fig. 2(a) are calculated from eqn (5) using the data in Fig. 2(c) and (d), and well capture the qualitative dependence of $K$ on $c_{0}$ obtained from our simulations. Fig. 2(c) and (d) illustrate how the protein-induced curvatures change the average separation and relative roughness of the adhering membranes. For $c_{0}^{(1)}=c_{0}^{(2)}= \pm c_{0}$ the protein-induced membrane curvatures render the average separation $\bar{l}$ of the two membranes away from the binding range, leading to a decrease in $K$ with $c_{0}$. And then an increase in membrane roughness $\xi_{\perp}$ is required for apposing membrane patches to locally reach the binding range via thermal fluctuations. For $-c_{0}^{(1)}=c_{0}^{(2)}=c_{0}$ the induced curvature hardly affects the average membrane separation, but suppresses the shape fluctuations of the membranes, causing a slight decrease in $\xi_{\perp}$.

The quantitative difference between theoretical prediction (dashed lines) and simulation data (filled symbols) at large $c_{0}$ in Fig. 2(a) implies that the curvature-induced protein-protein cis-repulsion neglected in eqn (4) affects the binding of receptors and ligands. Following the analytical solution $V(r)=8 \pi \kappa c_{0}^{2} \ell^{6} / r^{4}$ for the curvature-induced repulsion between two disks of radius $\ell$ embedded in a tensionless membrane ${ }^{29-32}$ we obtain with $c_{0}=0.05 \mathrm{~nm}^{-1}$ and $\ell \approx\left(a^{2} / \pi\right)^{1 / 2}=5.6 \mathrm{~nm}$ the estimate of $r_{0} \approx 15 \mathrm{~nm}$ at which $V\left(r_{0}\right)=k_{\mathrm{B}} T$. An order of magnitude increase in the numerical prefactor of $V(r)$ gives $r_{0} \approx 27 \mathrm{~nm}$, yet less than the average distance $\bar{d}=N_{\mathrm{R}}{ }^{-1 / 2} L_{x} \approx 42 \mathrm{~nm}$ between the receptors or ligands on either membrane. The seemingly weak cis-repulsion, however, does impact the binding as can be visualized from the pair correlation function $g(r)$ of the receptor-ligand complexes in Fig. 3. The transition from complex clustering to mutual exclusion, i.e., $g(r)>1$ to $g(r)<1$ clearly indicates the interplay between fluctuationinduced complex-complex attraction ${ }^{6,30}$ and curvature-induced protein-protein cis-repulsion. The attraction originates from the physical fact that the membranes can adopt more conformations when the complexes, which constrain the local membrane separations, get closer. At $-c_{0}^{(1)}=c_{0}^{(2)}=c_{0}$ or $c_{0}^{(1)}=$ $c_{0}^{(2)}= \pm 0.025 \mathrm{~nm}^{-1}$ the complex-complex attraction dominates, whereas the protein-protein cis-repulsion dominates at $c_{0}^{(1)}=c_{0}^{(2)}= \pm 0.05 \mathrm{~nm}^{-1}$, in accord with the fact that the cisrepulsion increases with $c_{0}$.

To understand the effect of curvature-induced cis-repulsion we introduce the binding constant of membrane-anchored

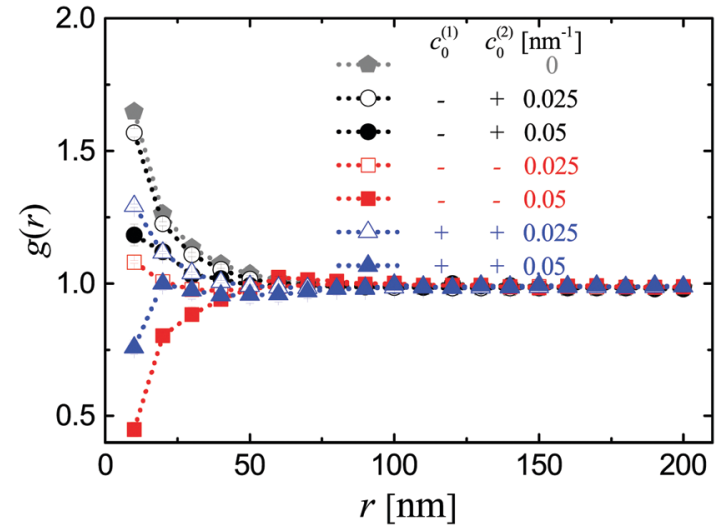

Fig. 3 Pair correlation function $g(r)$ of the receptor-ligand complexes obtained from the same simulations as in Fig. 2. The signs for the proteininduced membrane curvature $c_{0}^{(1)}$ and $c_{0}^{(2)}$ are explained in Fig. 1.

receptors and ligands

$$
K_{0}=\frac{n_{\mathrm{RL}} / A_{\mathrm{RL}}}{n_{\mathrm{R}} / A_{\mathrm{R}} \times n_{\mathrm{L}} / A_{\mathrm{L}}}
$$

by analogy to the standard description of thermodynamic equilibrium constant. The ratio of number of molecules $n_{\alpha}$ to effective area $A_{\alpha}$ in eqn (6) defines the effective concentration of each species $(\alpha=\mathrm{R}, \mathrm{L}$, or RL) and is linked to the thermodynamic activity $a_{\alpha}$ via $a_{\alpha}=\left(n_{\alpha} / A_{\alpha}\right) / c^{\ominus}$ with $c^{\ominus}$ the $2 \mathrm{D}$ analog of the standard concentration. $K_{0}$ is thus a constant irrespective of the overall protein concentration $[\alpha]_{0}$ or the protein-induced curvature $c_{0}$ if the membrane profile $P(l)$ in eqn (4) is somehow fixed, i.e., $K_{0}=\int K(l) P(l) \mathrm{d} l .{ }^{66}$ The apparent binding constant defined by eqn (1) is then

$$
\begin{gathered}
K=K_{0} \times \frac{A_{\mathrm{RL}} A}{A_{\mathrm{R}} A_{\mathrm{L}}} \\
=\frac{1}{2} K_{\mathrm{pl}}\left[\operatorname{Erf}\left(\tilde{l}_{1}\right)-\operatorname{Erf}\left(\tilde{l}_{2}\right)\right] \frac{A_{\mathrm{RL}} A}{A_{\mathrm{R}} A_{\mathrm{L}}} .
\end{gathered}
$$

Eqn (8) is obtained by inserting eqn (5) to the general result eqn (7). Now we turn to the estimation of effective area $A_{\alpha}$. Due to the protein-protein $c i s$-repulsion, receptors and ligands cannot move freely along the membranes with projected area of $A$, and behave as non-ideal 'solute' immersed in the membrane lipid 'solvent'. The 2D 'solution' of receptors $(\alpha=R)$ or ligands $(\alpha=\mathrm{L})$ can be described by the virial equation of state (EOS) $p_{\alpha} A /\left(N_{\alpha} k_{\mathrm{B}} T\right)=1+\sum_{n \geq 2} B_{n, \alpha}[\alpha]_{0}^{n-1}$ with $p_{\alpha}$ the $2 \mathrm{D}$ analog of pressure and $B_{n, \alpha}$ the $n$-th virial coefficient. Here we neglect the coupling between the two membranes due to receptorligand complex formation. By taking

$$
A_{\alpha}=\frac{A}{1+\sum_{n} B_{n, \alpha}[\alpha]_{0}^{n-1}},
$$

one recovers the EOS of the ideal gas $p_{\alpha} A_{\alpha} /\left(N_{\alpha} k_{\mathrm{B}} T\right)=1$, implying that the effective area $A_{\alpha}$ defined by eqn (9) is the translational area of the receptors or ligands as if they were regarded as ideal gases. Up to the second virial term eqn (9) reduces in the case of 
dilute solutions to $A_{\alpha}=A /\left(1+B_{2, \alpha}[\alpha]_{0}\right) \approx A\left(1-B_{2, \alpha}[\alpha]_{0}\right)$, the general formula for excluded-volume correction of $2 \mathrm{D}$ objects such as hard discs. Similarly, the effective area of the receptorligand complexes can be defined by eqn (9) with the concentration $[\mathrm{RL}]_{0}=\max \left(N_{\mathrm{R}}, N_{\mathrm{L}}\right) / A$, since the translation of the complexes is affected by both the receptors and ligands anchored to the apposing membranes. The calculation of virial coefficients $B_{n, \alpha}$ is provided in the Appendix. The solid lines in Fig. 2(a) and 4(a) are theoretical results calculated from eqn (8) with up to the fourth virial term and agree well with our simulation results from systems with different number of receptors and ligands. For systems with bending rigidity contrast between the protein-free and protein-containing membrane patches, the theoretical prediction exhibits systematic deviation from the simulation data as shown in Fig. 4(b) for $\kappa_{\mathrm{p}}=2 \kappa=20 k_{\mathrm{B}} T$. The underestimation arises from the fact that our theoretical account does not include the attraction between the stiffer protein-containing patches induced by membrane shape fluctuations. ${ }^{29,31}$ Nevertheless, our theory provides a good estimate for the binding constant of membraneanchored receptors and ligands that generate membrane curvatures.
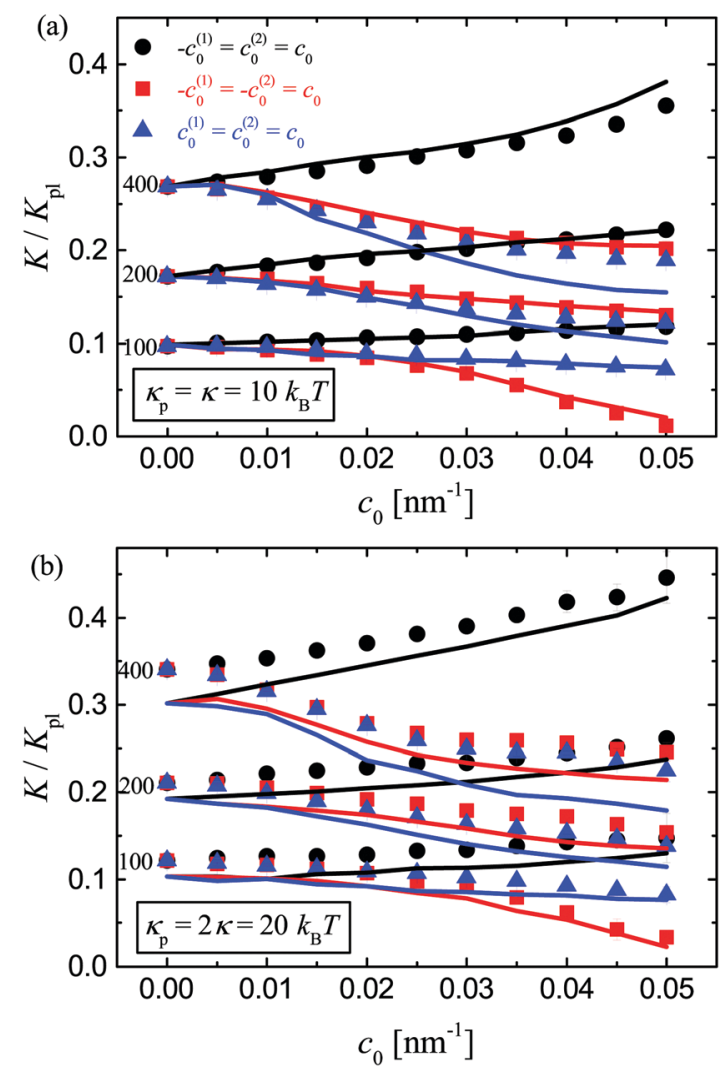

Fig. 4 Reduced receptor-ligand binding constant $K / K_{\mathrm{pl}}$ as a function of protein-induced membrane curvature $c_{0}$. The data points are from simulations with different number of receptors and ligands $\left(N_{R}=N_{L}=100,200\right.$, and 400 ). The bending rigidity of the protein-containing and protein-free membrane patches is (a) $\kappa_{\mathrm{p}}=\kappa=10 k_{\mathrm{B}} T$, and (b) $\kappa_{\mathrm{p}}=2 \kappa=20 k_{\mathrm{B}} T$. Solid lines are theoretical results from eqn (8) and correspond to data points in the same color.

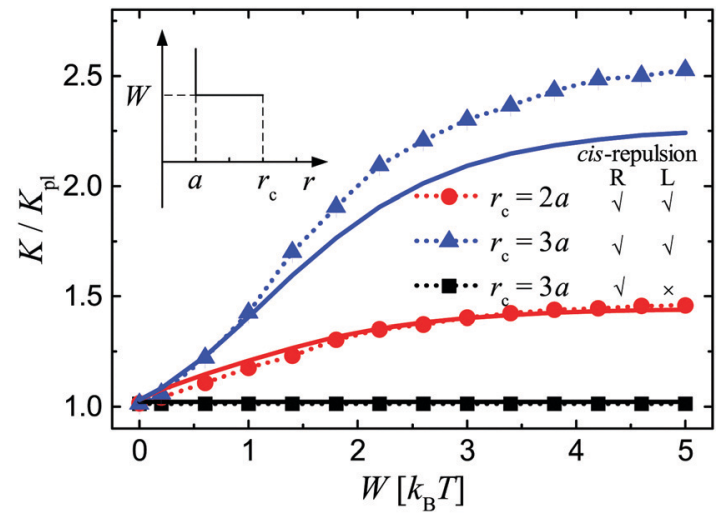

Fig. 5 Reduced receptor-ligand binding constant $K / K_{\mathrm{pl}}$ as a function of the strength $W$ of repulsive square-well potential between the proteins on each membrane. The data points are from simulations with $N_{R}=200$ receptors and $N_{\mathrm{L}}=200$ ligands anchored to planar membranes of area $60 a \times 60 a$. Solid lines are theoretical results from eqn (7) and correspond to data points in the same color.

As a further test we've simulated the binding of receptors and ligands that are anchored to planar membranes at separation within the binding range and experience cis-repulsion in the form of square-well potential. The estimates of binding constant from eqn (7) and the values directly measured from simulations agree within $10 \%$ as shown in Fig. 5 , confirming the effective area is a simple yet useful means for quantifying the effect of protein-protein cis-repulsion on the binding. Fig. 5 also shows for special cases where receptors or ligands do not experience cis-repulsion, i.e., $c_{0}^{(1)} c_{0}^{(2)}=0$, the binding constant remains unaffected by the cis-repulsion. This is due to the fact that the receptors or ligands can move freely along the anchoring membranes to find their binding partners. In these cases one can also easily obtain $A_{\mathrm{RL}} A /\left(A_{\mathrm{R}} A_{\mathrm{L}}\right)=1$ from eqn (9). ${ }^{67}$

\section{Conclusion and outlook}

We've investigated the binding of membrane-anchored receptor and ligand proteins that generate local membrane curvatures using MC simulations of a statistical mechanical model for membrane adhesion. We find that the local curvatures induced by receptors and ligands affect their binding by (i) altering the local separation and relative roughness of the two adhering membranes, and (ii) causing protein-protein cis-repulsion on each membrane. Depending on the signs of the curvatures, the binding constant increases or decreases with the curvatures. We provide a theory that incorporates the above two factors by taking into account (i) the distribution of local membrane separations, and (ii) the effective area for both unbound proteins and protein complexes to move freely along the membranes. The binding constant calculated from the theory is in good quantitative agreement with our simulation results.

Although our mesoscopic model does not include explicitly the molecular structure of the anchored proteins and rather describes the binding interaction via the simplified square-well potential, the main part of our theory as given by 
Table 1 The second to fourth virial coefficients for the membrane-anchored receptor and ligand proteins and receptor-ligand complexes. The curvatureinduced protein-protein cis-repulsion takes the form $V(r)=8 \pi \kappa c_{0}^{2} \ell^{6} / r^{4}$

\begin{tabular}{|c|c|c|c|c|c|c|}
\hline \multirow[b]{2}{*}{$\begin{array}{l}c_{0} \\
\left(\mathrm{~nm}^{-1}\right)\end{array}$} & \multicolumn{3}{|l|}{$\mathrm{R} / \mathrm{L}$} & \multicolumn{3}{|c|}{ R-L complex } \\
\hline & $\begin{array}{l}B_{2} \\
\left(\mathrm{~nm}^{2}\right)\end{array}$ & $B_{3}\left(\mathrm{~nm}^{4}\right)$ & $B_{4}\left(\mathrm{~nm}^{6}\right)$ & $\begin{array}{l}B_{2} \\
\left(\mathrm{~nm}^{2}\right)\end{array}$ & $B_{3}\left(\mathrm{~nm}^{4}\right)$ & $B_{4}\left(\mathrm{~nm}^{6}\right)$ \\
\hline 0.005 & 39.63 & 742.08 & 4678.93 & 56.05 & 1484.15 & 13234.0 \\
\hline 0.01 & 79.27 & 2968.31 & 37431.41 & 112.10 & 5936.61 & 105872.02 \\
\hline 0.015 & 118.90 & 6678.69 & 126331.02 & 168.15 & 13357.38 & 357318.07 \\
\hline 0.02 & 158.54 & 11873.22 & 299451.30 & 224.20 & 23746.44 & 846976.17 \\
\hline 0.025 & 198.17 & 18551.91 & 584865.82 & 280.25 & 37103.82 & 1654250.34 \\
\hline 0.03 & 237.80 & 26714.75 & 1010648.13 & 336.30 & 53429.50 & 2858544.58 \\
\hline 0.035 & 277.44 & 36361.74 & 1604871.80 & 392.35 & 72723.48 & 4539262.93 \\
\hline 0.04 & 317.07 & 47492.89 & 2395610.38 & 448.41 & 94985.77 & 6775809.38 \\
\hline 0.045 & 356.70 & 60108.18 & 3410937.44 & 504.46 & 120216.37 & 9647587.97 \\
\hline 0.05 & 396.34 & 74207.63 & 4678926.52 & 560.51 & 148415.27 & 13234002.70 \\
\hline
\end{tabular}

eqn (4), (6), (7) and (9) is general and can be applied to specific adhesion molecules via combination with detailed molecular modeling of the adhesion systems. It should also be noted that our theory is inapplicable to the binding of membrane-anchored receptors and ligands that undergo cis-attraction such as cadherin clustering ${ }^{7,68,69}$ and protein aggregation in lipid-raft domains, ${ }^{27}$ since the number of molecules changes upon localization and thereby the effective area defined in eqn (9) by itself is insufficient for the description of effective concentration.

Our study reveals that the ability to induce membrane curvatures represents molecular characteristics of the anchored receptors and ligands which affects their binding in cell adhesion and should be carefully considered in experimental characterization of the binding affinity.

\section{Conflicts of interest}

There are no conflicts to declare.

\section{Appendix}

We provide here the calculation of virial coefficients for membraneanchored receptors and ligands as well as the receptor-ligand complexes. The anchored proteins can be approximated as discs embedded in the 2D membranes. For the curvature-induced cisrepulsion $V(r)=8 \pi \kappa c_{0}^{2} \ell^{6} / r^{4}$, the second to fourth virial coefficients for receptor or ligand proteins are $B_{2}=2.7842 \mathscr{A}, B_{3}=3.662 \mathscr{A}^{2}$ and $B_{4}=1.622 \mathscr{A}^{3}$ with $\mathscr{A}=\ell^{2}\left(8 \pi \kappa c_{0}^{2} \ell^{2}\right)^{1 / 2}$ according to ref. 70 . For the receptor-ligand complexes, we use the repulsive potential $V(r)=$ $16 \pi \kappa c_{0}{ }^{2} \ell^{6} / r^{4}$ to calculate virial coefficients since both the bound receptors and ligands in the complexes experience cis-repulsion, and the corresponding values are $B_{2}=2.7842 \times \sqrt{2} \mathscr{A}, B_{3}=$ $3.662 \times(\sqrt{2} \mathscr{A})^{2}$ and $B_{4}=1.622 \times(\sqrt{2} \mathscr{A})^{3}$. Table 1 lists the virial coefficients for different values of the spontaneous curvature $c_{0}$.

For the square-well potential used in the test simulations for Fig. 5, the second and third virial coefficients are calculated according to the results in ref. 71 for two specific values of interaction range $r_{\mathrm{c}}=2 a$ and $3 a$. The coefficients are $B_{2}=b(1-3 f)$
Table 2 The second and third virial coefficients for the membraneanchored receptor and ligand proteins and receptor-ligand complexes. The protein-protein cis-repulsion assumes a square-well potential. The range of the potential is $r_{\mathrm{C}}=2 a$ or $3 a$, and strength $W=0-5 k_{\mathrm{B}} T$

\begin{tabular}{|c|c|c|c|c|c|}
\hline \multirow[b]{2}{*}{$r_{\mathrm{c}}$} & \multirow[b]{2}{*}{$W\left(k_{\mathrm{B}} T\right)$} & \multicolumn{2}{|l|}{$\underline{\mathrm{R} / \mathrm{L}}$} & \multicolumn{2}{|c|}{ R-L complex } \\
\hline & & $B_{2}\left(\mathrm{~nm}^{2}\right)$ & $B_{3}\left(\mathrm{~nm}^{4}\right)$ & $B_{2}\left(\mathrm{~nm}^{2}\right)$ & $B_{3}\left(\mathrm{~nm}^{4}\right)$ \\
\hline \multirow[t]{14}{*}{$2 a$} & 0 & 157.08 & 19295.19 & 157.08 & 19295.19 \\
\hline & 0.2 & 242.50 & 32801.58 & 312.44 & 54191.32 \\
\hline & 0.6 & 369.70 & 79762.60 & 486.38 & 157955.15 \\
\hline & 1.0 & 454.96 & 133174.91 & 564.54 & 232709.06 \\
\hline & 1.4 & 512.11 & 180439.66 & 599.66 & 272813.75 \\
\hline & 1.8 & 550.42 & 217755.86 & 615.44 & 292225.30 \\
\hline & 2.2 & 576.10 & 245446.74 & 622.53 & 301235.25 \\
\hline & 2.6 & 593.32 & 265255.27 & 625.72 & 305342.42 \\
\hline & 3.0 & 604.86 & 279106.75 & 627.15 & 307199.80 \\
\hline & 3.4 & 612.59 & 288653.32 & 627.79 & 308036.78 \\
\hline & 3.8 & 617.78 & 295171.33 & 628.08 & 308413.35 \\
\hline & 4.2 & 621.25 & 299594.22 & 628.21 & 308582.65 \\
\hline & 4.6 & 623.58 & 302583.21 & 628.27 & 308658.74 \\
\hline & 5.0 & 625.14 & 304597.72 & 628.30 & 308692.94 \\
\hline \multirow[t]{14}{*}{$3 a$} & 0 & 157.08 & 19295.19 & 157.08 & 19295.19 \\
\hline & 0.2 & 384.87 & 52415.95 & 571.37 & 132020.55 \\
\hline & 0.6 & 724.06 & 245391.39 & 1035.22 & 652338.63 \\
\hline & 1.0 & 951.43 & 516314.44 & 1243.65 & 1089169.45 \\
\hline & 1.4 & 1103.83 & 779970.80 & 1337.30 & 1336044.51 \\
\hline & 1.8 & 1206.0 & 999127.15 & 1379.38 & 1458058.50 \\
\hline & 2.2 & 1274.48 & 1166766.48 & 1398.29 & 1515200.14 \\
\hline & 2.6 & 1320.38 & 1288958.94 & 1406.78 & 1541350.54 \\
\hline & 3.0 & 1351.15 & 1375431.36 & 1410.60 & 1553197.23 \\
\hline & 3.4 & 1371.78 & 1435492.18 & 1412.32 & 1558539.84 \\
\hline & 3.8 & 1385.60 & 1476707.99 & 1413.09 & 1560944.38 \\
\hline & 4.2 & 1394.87 & 1504769.40 & 1413.43 & 1562025.61 \\
\hline & 4.6 & 1401.09 & 1523775.62 & 1413.59 & 1562511.60 \\
\hline & 5.0 & 1405.25 & 1536604.36 & 1413.66 & 1562730.00 \\
\hline
\end{tabular}

and $B_{3}=b^{2}\left(\frac{4}{3}-\frac{\sqrt{3}}{\pi}-1.65398 f+6.972 f^{2}-3.104 f^{3}\right)$ for $r_{\mathrm{c}}=2 a$, $B_{2}=b(1-8 f)$, and $B_{3}=b^{2}\left(\frac{4}{3}-\frac{\sqrt{3}}{\pi}-1.65398 f+25.2672 f^{2}\right.$ $\left.-35.6391 f^{3}\right)$ for $r_{\mathrm{c}}=3 a$. Here $b=\pi a^{2} / 2, f=\mathrm{e}^{-W / k_{\mathrm{B}} T}-1$ for receptor or ligand proteins and $f=\mathrm{e}^{-2 W / k_{\mathrm{B}} T}-1$ for the receptor-ligand complex. Table 2 lists the virial coefficients for potential strength $W=0-5 k_{\mathrm{B}} T$.

\section{Acknowledgements}

This work was supported by the Programs in the National Key Research and Development Program of China (Grant No. 2016YFA0501601), the National Natural Science Foundation of China (Grants No. 21504038 and 11472285) and the Strategic Priority Research Program of the Chinese Academy of Sciences (Grant No. XDB22040102). The numerical calculations in this paper have been done on the computing facilities in the High Performance Computing Center (HPCC) of Nanjing University.

\section{References}

1 B. Alberts, A. Johnson, J. Lewis, M. Raff, K. Roberts and P. Walter, Molecular Biology of the Cell, Garland Science, New York, 2014. 
2 B. W. Benham-Pyle, B. L. Pruitt and W. J. Nelson, Science, 2015, 348, 1024-1027.

3 G. Gaud, R. Lesourne and P. E. Love, Nat. Rev. Immunol., 2018, 18, 485-497.

4 G. I. Bell, M. Dembo and P. Bongrand, Biophys. J., 1984, 45, 1051-1064.

5 M. L. Dustin, S. K. Bromley, M. M. Davis and C. Zhu, Annu. Rev. Cell Dev. Biol., 2001, 17, 133-157.

6 H. Krobath, B. Różycki, R. Lipowsky and T. R. Weikl, Soft Matter, 2009, 5, 3354-3361.

7 Y. Wu, J. Vendome, L. Shapiro, A. Ben-Shaul and B. Honig, Nature, 2011, 475, 510-513.

8 T. P. Tolentino, J. Wu, V. I. Zarnitsyna, Y. Fang, M. L. Dustin and C. Zhu, Biophys. J., 2008, 95, 920-930.

9 J. B. Huppa, M. Axmann, M. A. Mörtelmaier, B. F. Lillemeier, E. W. Newell, M. Brameshuber, L. O. Klein, G. J. Schütz and M. M. Davis, Nature, 2011, 463, 963-967.

10 G. P. O'Donoghue, R. M. Pielak, A. A. Smoligovets, J. J. Lin and J. T. Groves, eLife, 2013, 2, e00778.

11 J. Steinkühler, B. Różycki, C. Alvey, R. Lipowsky, T. R. Weikl, R. Dimova and D. E. Discher, J. Cell Sci., 2019, 132, 216770.

12 D. J. Müller and Y. F. Dufrêne, Nat. Nanotechnol., 2008, 3, 261-269.

13 T. L. D. Le, Y. Guérardel, P. Loubière, M. Mercier-Bonin and E. Dague, Biophys. J., 2011, 101, 2843-2853.

14 M. Zhang, B. Wang and B. Xu, J. Phys. Chem. B, 2014, 118, 6714-6720.

15 J. Huang, V. I. Zarnitsyna, B. Liu, L. J. Edwards, N. Jiang, B. D. Evavold and C. Zhu, Nature, 2010, 464, 932-936.

16 B. Liu, W. Chen, B. D. Evavold and C. Zhu, Cell, 2014, 157, 357-368.

17 B. Liu, S. Zhong, K. Malecek, L. A. Johnson, S. A. Rosenberg, C. Zhu and M. Krogsgaard, Eur. J. Immunol, 2014, 44, 239-250.

18 C. D. Paschall, W. H. Guilford and M. B. Lawrence, Biophys. J., 2008, 94, 1034-1045.

19 L. Limozin, P. Bongrand and P. Robert, Sci. Rep., 2016, 6, 35193.

20 Q. Li, A. Wayman, J. Lin, Y. Fang, C. Zhu and J. Wu, Biophys. J., 2016, 111, 686-699.

21 J. Hu, G.-K. Xu, R. Lipowsky and T. R. Weikl, J. Chem. Phys., 2015, 143, 243137.

22 G.-K. Xu, J. Hu, R. Lipowsky and T. R. Weikl, J. Chem. Phys., 2015, 143, 243136.

23 G.-K. Xu, J. Qian and J. Hu, Soft Matter, 2016, 12, 4572-4583.

24 G.-K. Xu, Z. Liu, X.-Q. Feng and H. Gao, Phys. Rev. E, 2016, 93, 032411.

25 J. Hu, R. Lipowsky and T. R. Weikl, Proc. Natl. Acad. Sci. U. S. A., 2013, 110, 15283-15288.

26 L. Li, G.-K. Xu and F. Song, Phys. Rev. E, 2017, 95, 012403.

27 L. Li, J. Hu, X. Shi, Y. Shao and F. Song, Soft Matter, 2017, 13, 4294-4304.

28 L. Li, J. Hu, G. Xu and F. Song, Phys. Rev. E, 2018, 97, 012405.

29 M. Goulian, R. Bruinsma and P. Pincus, Europhys. Lett., 1993, 22, 145-150.

30 R. Bruinsma and P. Pincus, Curr. Opin. Solid State Mater. Sci., 1996, 1, 401-406.
31 J.-M. Park and T. C. Lubensky, J. Phys. I, 1996, 6, 1217-1235. 32 T. R. Weikl, M. M. Kozlov and W. Helfrich, Phys. Rev. E: Stat. Phys., Plasmas, Fluids, Relat. Interdiscip. Top., 1998, 57, 6988-6995.

33 G. G. Martins and J. Kolega, Cell Motil. Cytoskeleton, 2006, 63, 101-115.

34 G. Jiang, A. H. Huang, Y. Cai, M. Tanase and M. P. Sheetz, Biophys. J., 2006, 90, 1804-1809.

35 F. Lagarrigue, P. V. Anekal, H.-S. Lee, A. I. Bachir, J. N. Ablack, A. F. Horwitz and M. H. Ginsberg, Nat. Commun., 2015, 6, 8492.

36 T. A. Brown, T. Bouchard, T. Stjohn, E. Wayner and W. G. Carter, J. Cell Biol., 1991, 113, 207-221.

37 A. Avigdor, P. Goichberg, S. Shivtiel, A. Dar, A. Peled, S. Samira, O. Kollet, R. Hershkoviz, R. Alon, I. Hardan, H. Ben-Hur, D. Naor, A. Nagler and T. Lapidot, Blood, 2004, 103, 2981-2989. 38 H. T. McMahon and J. L. Gallop, Nature, 2005, 438, 590-596. 39 T. Baumgart, B. R. Capraro, C. Zhu and S. L. Das, Annu. Rev. Phys. Chem., 2011, 62, 483-506.

40 N. Ramakrishnan, P. B. S. Kumar and R. Radhakrishnan, Phys. Rep., 2014, 543, 1-60.

41 M. M. Kozlov, F. Campelo, N. Liska, L. V. Chernomordik, S. J. Marrink and H. T. McMahon, Curr. Opin. Cell Biol., 2014, 29, 53-60.

42 J. C. Stachowiak, E. M. Schmid, C. J. Ryan, H. S. Ann, D. Y. Sasaki, M. B. Sherman, P. L. Geissler, D. A. Fletcher and C. C. Hayden, Nat. Cell Biol., 2012, 14, 944-949.

43 B. Różycki and R. Lipowsky, J. Chem. Phys., 2015, 142, 054101.

44 B. Różycki and R. Lipowsky, J. Chem. Phys., 2016, 145, 074117.

45 G. J. Doherty and H. T. McMahon, Annu. Rev. Biochem., 2009, 78, 857-902.

46 H. T. McMahon and E. Boucrot, Nat. Rev. Mol. Cell Biol., 2011, 12, 517-533.

47 H. T. McMahon and E. Boucrot, J. Cell Sci., 2015, 128, 1065-1070.

48 P. Bassereau, R. Jin, T. Baumgart, M. Deserno, R. Dimova, V. A. Frolov, P. V. Bashkirov, H. Grubmüller, R. Jahn, H. J. Risselada, L. Johannes, M. M. Kozlov, R. Lipowsky, T. J. Pucadyil, W. F. Zeno, J. C. Stachowiak, D. Stamou, A. Breuer, L. Lauritsen, C. Simon, C. Sykes, G. A. Voth and T. R. Weikl, J. Phys. D: Appl. Phys., 2018, 51, 343001.

49 P. Sens, L. Johannes and P. Bassereau, Curr. Opin. Cell Biol., 2008, 20, 476-482.

50 R. Lipowsky, Faraday Discuss., 2013, 161, 305-331.

51 Y. Liu, J. Agudo-Canalejo, A. Grafmüller, R. Dimova and R. Lipowsky, ACS Nano, 2016, 10, 463-474.

52 J. Mao, P. Chen, J. Liang, R. Guo and L.-T. Yan, ACS Nano, 2016, 10, 1493-1502.

53 P. Chen, Z. Huang, J. Liang, T. Cui, X. Zhang, B. Miao and L.-T. Yan, ACS Nano, 2016, 10, 11541-11547.

54 H. Noguchi, Soft Matter, 2017, 13, 7771-7779.

55 T. R. Weikl and R. Lipowsky, Biophys. J., 2004, 87, 3665-3678. 56 B. Różycki, T. R. Weikl and R. Lipowsky, Phys. Rev. Lett., 2008, 100, 098103.

57 L. Li, X. Wang, Y. Shao, W. Li and F. Song, Sci. China: Phys., Mech. Astron., 2018, 61, 128711. 
58 T. R. Weikl and R. Lipowsky, Membrane adhesion and domain formation, in Advances in Planar Lipid Bilayers and Liposomes, ed. A. Leitmannova Liu, Academic Press, Amsterdam, 2006, ch. 4.

59 W. T. Góźdź and G. Gompper, Europhys. Lett., 2001, 55, 587-593.

60 P. K. Tsourkas, M. L. Longo and S. Raychaudhuri, Biophys. J., 2008, 95, 1118-1125.

61 J. Zimmerberg and M. M. Kozlov, Nat. Rev. Mol. Cell Biol., 2006, 7, 9-19.

62 D. Marsh, Biophys. J., 2007, 93, 3884-3899.

63 R. P. Bradley and R. Radhakrishnan, Proc. Natl. Acad. Sci. U. S. A., 2016, 113, E5117-E5124.

64 M. L. Dustin, L. M. Ferguson, P.-Y. Chan, T. A. Springer and D. E. Golan, J. Cell Biol., 1996, 132, 465-474.

65 A. Grakoui, S. K. Bromley, C. Sumen, M. M. Davis, A. S. Shaw, P. M. Allen and M. L. Dustin, Science, 1999, 285, 221-227.
66 This is analogous to the standard thermodynamic equilibrium constant which is defined via the ratio of activities and independent of the concentrations of reactants and products.

67 A. S. Yap, W. M. Brieher, M. Pruschy and B. M. Gumbiner, Curr. Biol., 1997, 7, 308-315.

68 For ligands without cis-repulsion, $A_{\mathrm{RL}}=A_{\mathrm{R}}$ and $\lim _{\rho \rightarrow 0} A_{\mathrm{L}}=A$. Similar relations hold for receptors without cis-repulsion. The reduced binding constant in Fig. 5 is not strictly equal to 1 because the short-range receptor-receptor repulsion cannot be neglected at finite concentration.

69 W. Baumgartner, G. J. Schütz, J. Wiegand, N. Golenhofen and D. Drenckhahn, J. Cell Sci., 2003, 116, 1001-1011.

70 J. G. Briano and E. D. Glandt, Fluid Phase Equilib., 1981, 6, 275-277.

71 N. A. R. Hussein and S. M. Ahmed, J. Phys. A: Math. Gen., 1991, 24, 289-305. 